\title{
Phase Gratings For The Sub-Millimetre Band
}

\author{
R. May, J.A. Murphy, W. Lanigan \\ Department of Experimental Physics, NUI Maynooth, Ireland
}

\begin{abstract}
This presentation is concerned with the development of phase gratings for use at Terahertz frequencies (sub-mm wavelengths). Design, fabrication and testing of two grating types (Dammann and Fourier) are discussed. Modelling using quasi-optical techniques is outlined and the implementation of a suitable algorithm (Gerchberg-Saxton) to find solutions for the phase-retrieval problem discussed.
\end{abstract}

\section{Introduction}

Only in recent years has terahertz or T-Ray technology become a practical area as electronics research reduces the size and cost of T-Ray sources and detectors. With possible future imaging applications in areas such as security, nondestructive materials testing and pharmaceuticals quality control, a drive is on to develop single- and multi-pixel imaging systems. Any imaging system requires optical components (lenses, mirrors, etc.) to focus and redirect beams. A common requirement of an imaging system is the ability to split and shape a beam. The phase grating provides an elegant, more efficient alternative to the traditional bearn splitter. In a heterodyne array receiver system, for example a phase grating can be used to match the signal beam of a single local oscillator source to an array of detector devices. Used in this way a phase grating can be referred to as a multiplexing device. Operated in reverse the phase grating can be used to combine several coherent beams into a single powerful beam $^{[1]}$. Presented here are the results of numerical modelling of two types of phase gratings: Dammann and Fourier, followed by measurements of the output patterns generated by gratings manufactured for use at frequencies of $0.1 \mathrm{THz}$.

Unlike components designed for use at optical wavelengths, where the size of the components is much greater than the wavelengths used, in the terahertz region the size of components is comparable to that of the wavelength and therefore diffraction becomes an important consideration. Because of the significance of diffractive effects such components are referred to as diffractive optical elements. Since geometrical optics techniques are insufficient to describe effects due to diffraction, quasi-optical techniques such as Fresnel and Fraunhofer diffraction must be used. The phase gratings examined in this paper were modelled using these methods.

\section{Basic Theory of Phase Gratings}

Whereas the diffraction grating modulates both amplitude and phase, the phase grating modulates phase only and therefore has higher throughput of power - an important consideration at long wavelength optics.

According to the Array Theorem ${ }^{[2]}$ the output generated by a diffraction grating $E(u, v)$ is the combination of the interference due to a linear array of cells and the diffraction pattern generated by a single cell

$$
E(u, v)=|T(u, v)| \cdot|A(u, v)|
$$

where - indicates multiplication. The two terms on the right can be treated independently: the interference term $A(u, v)$ determines the separation of the diffraction orders; the diffraction term $T(u, v)$ controls the ratio of power in each diffraction order. By suitable manipulation of the diffraction term, one can direct the incident power into a specific set of diffraction orders.

The diffraction term is determined by the transmission function $t_{\text {cell }}(x, y)$ which is simply a mathematical function describing the phase profile imposed on the incident beam by the cell. The general form of the transmission function for a single cell is

$$
t_{\text {cell }}(x, y)=\exp \left( \pm i \phi_{\text {cell }}(x, y)\right)
$$

where $\phi_{\text {cell }}(x, y)$ describes the phase profile of the cell. If the grating is illuminated with a quasi-Gaussian beam that has a flat phase-front then the only phase structure in the plane of the grating is that due to the phase modulation of the grating itself. Therefore the problem of finding a phase grating that is able to produce a given output $E(u, v)$ is reduced to finding $\phi_{\text {cell }}(x, y)$.

Broadly speaking phase gratings can be divided into two classes: discrete-level phase gratings and Fourier (continuous phase) gratings. These are discussed next.

\section{Discrete-level phase gratings}

In discrete-level gratings the continuous phase profile required to produce a certain output is approximated with a finite number of discrete phase levels (with values in the range 0 to $2 \pi$ ). The points at which the phase jumps between allowed phase levels are called transition points. The task of finding a solution is thus reduced to finding a suitable set of transition points using a set number of phase levels that will generate the required output diffraction pattern. The simplest, if not the most elegant way of finding a solution set of transition points is to use a brute force method whereby all values for transition points along the length of the unit cell are tested, and the set resulting in the highest efficiency chosen. A useful tool in grating design is the incorporation of symmetries (reflection or translational or both) into the grating pattern, which can reduce significantly the design complexity ${ }^{[3]}$. Within this class of grating, individual gratings can be characterized by properties they possess: the number of phase levels, different symmetries imposed upon the grating pattern, etc.

One important type of discrete-level grating design is the Dammann grating (DG), so-called in recognition of the early work done by Dammann on this subject ${ }^{[4],[5]}$. This is a binary level grating, i.e. possessing only two phase levels (usually chosen to be 0 and $\pi$ ). The number of degrees of freedom for a DG is then simply equal to the number of transition points per unit cell. The main simplification in Dammann's method is the restriction of the two-dimensional transmission function to only those separable into two dimensions, i.e. $t_{\text {cell }}\left(x_{y} y\right)=t_{1}(x) \bullet$ $t_{2}(y)$, thereby reducing the complexity by changing it to a onedimensional problem. Obviously this reduces the applicability of this solution since output patterns inseparable into two dimensions can never be achieved with a DG.

\section{Fourier Grating}

Phase gratings with a continuous phase profile are often referred to as Fourier gratings and can generate patterns with very high efficiencies. The problem of finding solutions for 
Fourier gratings requires a different approach than the one discussed above. One method, based on the Phase-Retrieval problem is the Gerchberg-Saxton Algorithm ${ }^{[6]}$. An iterative algorithm, its goal is to determine the phase required at the grating to produce the desired far-field amplitude distribution, $E_{\mathrm{F}}(u, v)$, given the amplitude distribution at the grating plane, $E_{\mathrm{G}}(x, y)$. The advantage of this method over Dammann's is that transmission functions that are inseparable into two dimensions can be found.

\section{Fabrication}

For transmission gratings, the phase profile is produced by making use of the phase delay imposed upon an incident field by changes in refractive index of the material through which it propagates. A simple method used to achieve this is to make the grating from a transparent material of uniform refractive index into which grooves of different depths can be milled. It is the difference in refractive indices, $\Delta n$, between the grating material and the surrounding medium (usually air), combined with the depth of material through which the incident field must pass that determines the phase delay. For reflection gratings, a phase delay is achieved by the fact that at each point on a groove the incident wave will be delayed at a deep part because the wave will have further to travel than at a shallow part. Whereas the maximum groove depth (corresponding to a phase delay of $\pi$ ) for a transmission grating is equal to $\lambda$, in reflection this becomes $\lambda / 2$. The phase grating, consisting of a regular array of slots or grooves, can be milled into a material of suitable refractive index (transmission grating) or reflective material (reflection grating).

While transmsission gratings have high throughput, losses can be incurred due to the presence of standing waves. On the other hand while reflection gratings do not suffer from standing waves, they must be designed with either off-axis input or output or both. For discrete-phase level gratings, a transmission design is favourable, since a reflective design would result in severe shadowing effects (truncation) in the sharp concave comers of the grating profile. Since no such sharp corners would be present in a Fourier grating profile, this type of grating should be designed in reflection to take advantage not only of the absence of standing waves, but also of the relative ease of milling the profile into a reflective block of aluminium rather than a plastic material (such as HDPE with a refractive index of 1.52 that could be used in transmission).

It was decided to manufacture and test two gratings: a transmission DG and a reflection Fourier grating. The gratings were machined on a CNC milling machine. The cutting tools used have a minimum radius of curvature which restricts the accuracy with which features can be milled, such as the concave comers present in a DG. The minimum radius of curvature available for machining the gratings easily in the department workshop is $1 \mathrm{~mm}$. The effect of this machining limit has been modelled for each of the gratings to test how adversely it affects the performance of each grating.

\section{Numerical and Experimental Results}

Examples of two of the above grating types were modelled, machined and tested.

A DG to create an on-axis array of $3 \times 3$ beams was machined as a transmission grating from a piece of HDPE (Fig.1). By using Dammann's technique, a one-dimensional transmission function $t_{1}(x)$ to create 3 beams in the $x$ dimension was calculated. The diffraction efficiency $\eta$ for this grating is low at $\sim 44 \%(66.4 \%$ in each dimension), which is due to the unsuccessful suppression of $2^{\text {nd }}$ order diffraction beams.
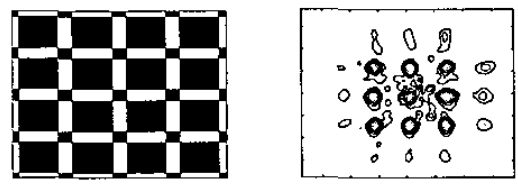

Fig.1: DG profile (left) and 3×3output (right)

The other grating made was a reflection Fourier grating, machined from a sheet of free-cutting aluminium and designed to create an off-axis array of $3 \times 1$ beams with a $38^{\circ}$ angle of throw. Fig. 2 shows expected and actual outputs from the grating (the central beam is at $38^{\circ}$ to gratings normal). An advantage of the reflection grating is that it allows for use of a much more compact imaging system than the one required for a transmission grating which requires an in-line 4-f imaging system
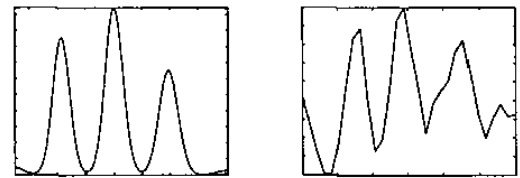

Fig.2: Fourier grating output: expected (left) and experimental (right)

\section{Conclusion}

Initial tests of both gratings in the laboratory indicate that the two grating types discussed here provide suitable methods of beam multiplexing at sub-mm wavelengths. Future work will concentrate on the use of other phase-retrieval methods, other than the GS one used here for the design, manufacture and testing of more sophisticated gratings.

\section{References}

[1] Shahabadi M. and Schunemann K., Proceedings of $2^{\text {nd }}$ ESA Workshop on Millimetre Wave Technology and Applications: Antennas, Circuits and Systems, p275-280, May 1998

[2]Murphy J.A., Withington S., van der Stadt H, ASP Conf. Ser. 75, D.T. Emerson, J.M. Payne, ASP, p238-244, 1995

[3] Morrison R.L., Opt. Soc. Am., vol. 9, p464-471 1992

[4] Dammann H., Gortler K., Optics Communications, vol. 3, No. 5, p312-315, July 1971

[5] Dammann H. and Klotz E., Optica Acta, vol. 24, No. 8, p505-515, 1977

[6] Gerchberg R.W. and Saxton W.O., Optik 35, p237-246, 1972 